\title{
AN ANALYTICAL APPROACH TO PREDICTION OF INTERNAL DEFECTS DURING THE FLAT ROLLING PROCESS OF STRAIN-HARDENING MATERIALS
}

\author{
Parvaneh Amjadian, Heshmatollah Haghighat \\ Razi University, Mechanical Engineering Department, Kermanshah, Iran \\ e-mail: hhaghighat@razi.ac.ir
}

\begin{abstract}
In this paper, the upper bound method has been used in analysis of the flat rolling process and prediction of internal defects for a strain-hardening material. The arc of contact has been replaced by a chord. The inlet shear boundary of the deformation zone has been assumed as an exponential curve and the boundary at the exit has been assumed as a cylindrical surface. A kinematically admissible velocity field has been proposed and internal, shear and frictional power terms have been derived. By minimizing the total power with respect to the neutral point position and the shape of the inlet shear boundary, the rolling torque has been determined. A criterion has been presented to predict the occurrence of the internal defects for given rolling conditions. Comparison of the analytically developed approach for rolling torque and internal defects with published theoretical and experimental data shows generally good agreement.
\end{abstract}

Keywords: upper bound, flat rolling, internal defects, strain-hardening exponent

\section{Introduction}

Flat rolling is the process of reducing thickness of a long sheet by torques applied through a set of rolls. The rolling process widely is used in production of industrial components, so concentrating on the final quality of the parts produced by this process is very important. Internal central bursts are common rolling processing defects. Ignoring their prediction causes both losses of physical injury and property damage or reducing quality of the final products. Usually, it is very difficult to detect central bursting defects by surface inspection. The investigations on flat rolling have been conducted for decades and various aspects of these processes have been studied. Dyja and Pietrzyk (1983) analyzed an asymmetric dual hot-rolled sheet using minimum energy, and replaced the arc of contact by a chord. Avitzur et al. (1988) modeled the flat rolling process by using upper bound analysis for rigid perfectly plastic materials to predict internal defects. Takuda et al. (1989) analyzed the strip rolling process by assuming a simple velocity field by considering free deformation zones in front and behind the roll gap. Turczyn and Pietrzyk (1992) analyzed the effect of deformation zone geometry on the internal defect in the flat rolling process. They used the upper bound method and took into consideration velocity boundaries. Discontinuity circular arcs were present and uniform velocity fields for internal defects assumed. Prakash and Dixit (1995) proposed a model for steady state plane strain cold rolling of a strain-hardening material which could predict the roll force and torque with reasonable engineering accuracy over the usual range of process variables. Turczyn (1996) analyzed the effect of deformation zone geometry on the internal defects in rolling process using the upper bound method. Martins and Barata (1999) presented an approach for analyzing plane strain rolling. They used the upper bound method to estimate the rolling torque and to model the material flow within the region of deformation between the rolls. Dogruoglu (2001) introduced a 
systematic method for constructing kinematically admissible velocity fields, which was necessary in the analysis of the plastic forming process by the upper bound method. Gosh and Gardiner (2004) identified various modeling issues that were necessary for successful simulation of the cold rolling process by comparing it with experiments on aluminum alloys. Rajak and Reddy (2005) analyzed internal defects in the plane strain rolling process with the finite element method. They used critical damage criteria. Serajzadeh and Mahmoodkhani (2008) presented a combined upper bound and finite element model for prediction of velocity and temperature fields during the hot rolling process. The closing behavior of internal defects in the central part of a continuous casting steel slab during rough transverse rolling was investigated by using FE-code, ANSYS/LS-DYNA by Deng et al. (2009). Misicko et al. (2009) observed and analyzed by computer simulation behavior of artificial surface defects inside flat steel samples during the hot rolling process by selected parameters. Cao et al. (2015) investigated the prediction of damage for the ultimate wire flat rolling process of high carbon steel using three different approaches of ductile damage. Haghighat and Saadati (2015) presented the kinematically admissible velocity field for the rolling of sandwich sheets, non-bonded before rolling, and discussed some mathematical aspects by the upper bound method. Haghighat and Pargzadeh (2017) investigated the effect of strain hardening on the central bursting defects in rod extrusion process. In this paper, analyses of plastic deformation and prediction of internal defects in flat rolling process of a strain-hardening material using the upper bound method is considered. The arc of contact between the roll and the sheet is replaced by a chord. Based on the FEM, it is observed that the inlet and outlet shear boundaries are not circular and in any condition such as inlet thickness and reduction in area are deferent, then we present arbitrary exponential shear boundaries and velocity field that can predict rolling torque and internal defects. Then a kinematically admissible velocity field is proposed and internal, shear and frictional power terms are derived. By minimizing the total power with respect to the neutral point position and the shape of the inlet shear boundary, the rolling torque and the occurrence of internal defects is investigated. The effect of the friction factor, strain-hardening exponent and reduction in the area on the rolling torque and safe and unsafe domains are investigated.

\section{Upper bound analysis}

Figure 1 shows flat rolling process parameters in a schematic diagram. Because of symmetry of the process, only half of the section is considered. An important subject in the upper bound analysis is the assumption of shear boundaries and a kinematically admissible velocity field satisfying volume constancy in the deformation zone and boundary conditions. In order to determine the velocity field, the arc of contact is replaced by a chord. The material starts as a sheet of thickness $t_{i}$ and then is deformed into a sheet of thickness $2 t_{f}, v_{i}$ is the initial velocity of the sheet and $v_{f}$ is the velocity of the product, $\alpha$ is the angle of the line connecting the initial point to the final point of the contact arc with the axis of symmetry. $R$ is radius, and $\omega$ is angular velocity of the roll.

\subsection{Velocity zones}

To analyze the process, the material under deformation is divided into three deformation zones, as shown in Fig. 1. In zones I and III, the material moves rigidly with the velocity $v_{i}$ and $v_{f}$, respectively. Zone II is the deformation zone and is surrounded by two shear surfaces $S_{1}$ and $S_{2}$ and the contact surface $S_{3}$. The shear boundary $S_{2}$ at the exit from the deformation zone is assumed to be a cylindrical surface with its center at the apex $O$. The shear boundary $S_{1}$ 


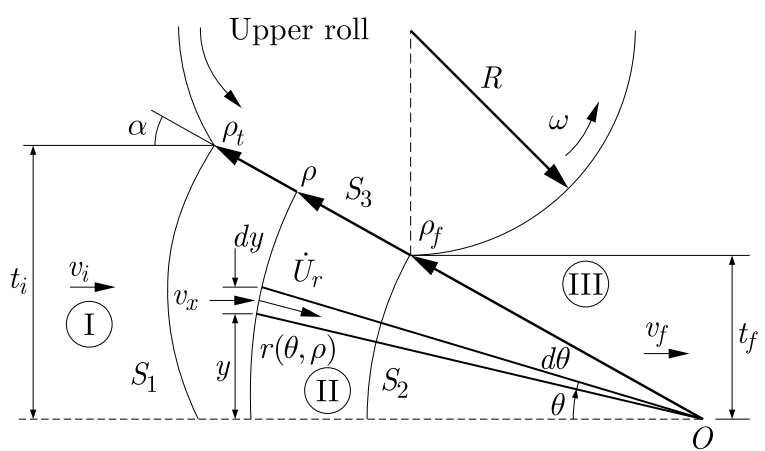

Fig. 1. Geometry of the sheet, roll, deformation zone and the shear boundaries

is assumed to be an arbitrarily curved surface. Using this description, mathematical equations of the shear boundaries $S_{1}$ and $S_{2}$ are defined in the cylindrical coordinates by

$$
r_{i}\left(\theta, \rho_{i}\right)=\rho_{i} \exp \left[\frac{b(\theta-\alpha)}{\alpha}\right] \quad r_{f}\left(\theta, \rho_{f}\right)=\rho_{f}
$$

where $r_{i}$ and $r_{f}$ are the radial position of inlet and outlet shear boundary, respectively, and the radial position in the deformation zone is

$$
r(\theta, \rho)=\rho \exp \left[\frac{b(\theta-\alpha)}{\alpha} \frac{\rho-\rho_{f}}{\rho_{i}-\rho_{f}}\right]=\rho g(\theta, \rho)
$$

where $\rho_{i}$ and $\rho_{f}$ are the radial distances from the virtual apex to inlet and outlet shear boundaries on the roll surface shown in Fig. $1, b$ is the shape factor of the shear boundary at the inlet of deformation zone II, $\theta$ is the peripheral position and $\rho$ is the radial position on the contact surface, and $g$ is an arbitrary shape function

$$
g(\theta, \rho)=\exp \left[\frac{b(\theta-\alpha)}{\alpha} \frac{\rho-\rho_{f}}{\rho_{i}-\rho_{f}}\right]
$$

$b$ can be negative, zero or positive. When $b$ is negative, the shear boundary moves away from the origin $O$, when $b$ is positive the shear boundary moves towards the apex $O$, when $b$ is zero, the inlet shear boundary is a cylindrical surface (i.e. $g=1$ ). In zone I and III, the material moves as a rigid body in the axial direction. In zone III, the velocity $v_{f}$ from the volume flow balance is

$$
v_{f}=v_{i} \frac{\rho_{i}}{\rho_{f}}
$$

With regard to the equilibrium of volume flow, the admissible velocity filed in deformation zone II can be obtained as

$$
v_{x}(d y)=-\dot{U}_{r}(r d \theta)
$$

$v_{x}$ is the horizontal velocity component in the deformation zone, $\dot{U}_{r}$ is the radial component of velocity and $y$ is the vertical position.

So, the radial velocity component of the velocity field is

$$
\dot{U}_{r}=-\frac{v_{x}}{r} \frac{d y}{d \theta}=-v_{i} \frac{\rho_{i}}{\rho}\left(\cos \theta+\frac{1}{g} \frac{\partial g}{\partial \theta} \sin \theta\right)
$$

The volume constancy in the cylindrical coordinates system is defined as

$$
\operatorname{dot}_{r r}+\dot{\varepsilon}_{\theta \theta}+\dot{\varepsilon}_{z z}=0
$$

where $\dot{\varepsilon}_{r r}, \dot{\varepsilon}_{\theta \theta}, \dot{\varepsilon}_{z z}$ are normal strain rate components. 
According to the cylindrical coordinates strain rates components and the assumption of the plane strain process, $\dot{U}_{z}=0$ (lateral velocity component), the peripheral velocity component $\left(\dot{U}_{\theta}\right)$ is

$$
\dot{U}_{\theta}=v_{i} \rho_{i} \frac{\partial g}{\partial r} \sin \theta
$$

where

$$
\frac{\partial g}{\partial r}=\frac{\partial g}{\partial \rho} \frac{\partial \rho}{\partial r}=\frac{b(\theta-\alpha)}{\alpha} \frac{1}{\rho_{i}-\rho_{f}} \frac{1}{1+\frac{b(\theta-\alpha)}{\alpha} \frac{\rho}{\rho_{i}-\rho_{f}}}
$$

The velocity components in deformation zone II are given as

$$
\dot{U}_{r}=-v_{i} \frac{\rho_{i}}{\rho}\left(\cos \theta+\frac{1}{g} \frac{\partial g}{\partial \theta} \sin \theta\right) \quad \dot{U}_{\theta}=v_{i} \rho_{i} \frac{\partial g}{\partial r} \sin \theta \quad \dot{U}_{z}=0
$$

As it is clear from Eqs. (2.10), on the axis of symmetry $\dot{U}_{\theta}=0$, and on the contact surface between the roll and sheet $\dot{U}_{\theta}=0$, so the incompressibility condition is satisfied. Nonzero strain rate components in the deformation zone are

$$
\begin{aligned}
& \dot{\varepsilon}_{r r}=v_{i} \frac{\rho_{i}}{\rho^{2}} \frac{1}{g}\left[\left(1-\rho \frac{\partial g}{\partial r}\right) \cos \theta+\left(\frac{1}{g} \frac{\partial g}{\partial \theta}-\rho \frac{\partial^{2} g}{\partial r \partial \theta}\right) \sin \theta\right] \\
& \dot{\varepsilon}_{\theta \theta}=-v_{i} \frac{\rho_{i}}{\rho^{2}} \frac{1}{g}\left[\left(1-\rho \frac{\partial g}{\partial r}\right) \cos \theta+\left(\frac{1}{g} \frac{\partial g}{\partial \theta}-\rho \frac{\partial^{2} g}{\partial r \partial \theta}\right) \sin \theta\right] \\
& \dot{\varepsilon}_{r \theta}=\frac{1}{2} v_{i} \frac{\rho_{i}}{\rho^{2}} \frac{1}{g}\left\{\left[\rho^{2} \frac{\partial^{2} g}{\partial r^{2}}+\frac{1}{g^{2}}\left(\frac{\partial g}{\partial \theta}\right)^{2}-\frac{1}{g} \frac{\partial^{2} g}{\partial \theta^{2}}+1\right] \sin \theta+\frac{1}{g} \frac{\partial g}{\partial \theta} \cos \theta\right\}
\end{aligned}
$$

\subsection{Determinations of power terms}

The internal power in the upper bound analysis for a perfectly elasic von Mises material in the deformation zone is

$$
\dot{W}_{i}=\frac{2}{\sqrt{3}} \sigma_{0} \int_{V} \sqrt{\frac{1}{2} \dot{\varepsilon}_{i j} \dot{\varepsilon}_{i j}} d V
$$

where $\dot{W}_{i}$ is internal power of deformation, $\sigma_{0}$ is the average flow stress and $d V$ is a differential volume of the deformation zone.

After substitution and simplification, the internal power of region II is given by

$$
\dot{W}_{i}=\frac{2 \sigma_{0}}{\sqrt{3}} \int_{0}^{\alpha} \int_{\rho_{f}}^{\rho_{i}} \sqrt{\dot{\varepsilon}_{r r}^{2}+\dot{\varepsilon}_{r \theta}^{2}} \rho g\left(g+\rho \frac{\partial g}{\partial \rho}\right) d \rho d \theta
$$

The shear power loss at the shear boundary is

$$
\dot{W}_{S}=\frac{\sigma_{0}}{\sqrt{3}} \int_{S_{1}, S_{2}}|\Delta v| d S
$$

where $\dot{W}_{S}$ is the shear power loss along the shear boundary, $\Delta v$ is the velocity difference and $d S_{1}, d S_{2}$ are differential areas of shear surfaces. For calculation of the power consumption on 
each surface of velocity discontinuity, the area of discontinuity and the amount of velocity discontinuity must be determined. With attention to Fig. 1

$$
\begin{aligned}
& d S_{1}=\rho_{i} g\left(\theta, \rho_{i}\right) \sqrt{1+\left(\frac{b}{\alpha}\right)^{2}} d \theta \\
& \left|\Delta v_{1}\right|=\left|v_{i}\left(1-\rho_{i} \frac{\partial g}{\partial r} \frac{1}{1+(b / \alpha)^{2}}\right) \sqrt{1+\left(\frac{b}{\alpha}\right)} \sin \theta\right|
\end{aligned}
$$

thus the shear power loss on the shear surface $S_{1}$ is obtained as

$$
\dot{W}_{S_{1}}=\frac{\sigma_{0}}{\sqrt{3}} v_{i} \rho_{i}\left[1+\left(\frac{b}{\alpha}\right)^{2}\right] \int_{0}^{\alpha} g\left(\theta, \rho_{i}\right)\left|1-\rho_{i} \frac{\partial g}{\partial r} \frac{1}{1+(b / \alpha)^{2}}\right| \sin \theta d \theta
$$

also for the shear surface $S_{2}$, the differential area and the amount of velocity discontinuity can be obtained respectively by

$$
d S_{2}=\rho_{f} d \theta \quad\left|\Delta v_{2}\right|=\left|v_{f} \sin \theta\right|
$$

the shear power loss on the shear surface $S_{2}$ is obtained as

$$
\dot{W}_{S_{2}}=\frac{\sigma_{0}}{\sqrt{3}} v_{f} \rho_{f} \int_{0}^{\alpha} \sin \theta d \theta
$$

The friction power loss at the interface of the sheet and the roll in $S_{3}$ and its general relation is

$$
\begin{aligned}
& \dot{W}_{f}=\frac{m \sigma_{0}}{\sqrt{3}} \int_{S_{3}}|\Delta v| d S \quad d S_{3}=d \rho \\
& \Delta v=\left|\dot{U}_{r}\right|_{\theta=\alpha}+R \omega|=|-v_{i} \frac{\rho_{i}}{\rho}\left(\cos \alpha+\frac{b}{\alpha} \frac{\rho-\rho_{f}}{\rho_{i}-\rho_{f}} \sin \alpha\right)+R \omega \mid
\end{aligned}
$$

where $\dot{W}_{f}$ is the friction power loss, $d S_{3}$ is a differential area of the frictional surface and $m$ is the friction factor after simplification

$$
\dot{W}_{f}=m \frac{\sigma_{0}}{\sqrt{3}} v_{i} \rho_{i} \int_{\rho_{f}}^{\rho_{i}} \frac{1}{\rho}\left|\cos \alpha+\frac{b}{\alpha} \frac{\rho-\rho_{f}}{\rho_{i}-\rho_{f}} \sin \alpha-\frac{R \omega \rho}{v_{i} \rho_{i}}\right| d \rho
$$

In the rolling process, there is only one point along the surface of contact between the roll and the sheet at which the surface velocity of the roll equals to the velocity of the sheet. This point is called the neutral point. Between the inlet shear surface and the neutral point the sheet is moving slower than the roll surface and on the exit side of the neutral point, the sheet moves faster than the roll surface. The position of the neutral point is where the relative velocity is zero, then

$$
v_{i} \frac{\rho_{i}}{\rho_{N}}\left(\cos \alpha+\frac{b}{\alpha} \frac{\rho_{N}-\rho_{f}}{\rho_{i}-\rho_{f}} \sin \alpha\right)-R \omega=0
$$

where $\rho_{N}$ is the radial position of the neutral point on the contact surface. 


\subsection{Effective flow stress}

Since actual metals exhibit strain-hardening behavior and the upper bound method is based on the assumption of perfectly elastic material, amendments must be made to match the theoretical and actual behavior. For a plastic material, the mean flow stress of the material $\sigma_{0}$ is given by

$$
\sigma_{0}=\frac{1}{\bar{\varepsilon}} \int_{0}^{\bar{\varepsilon}} \bar{\sigma} d \bar{\varepsilon} \quad \bar{\varepsilon}=\frac{2}{\sqrt{3}} \ln \frac{t_{i}}{t_{f}}
$$

In this paper, a modified upper bound method is used and behavior of the material is considered as

$$
\bar{\sigma}=K(\bar{\varepsilon})^{n}
$$

where $K$ is the strength coefficient, $n$ is the strain-hardening exponent, $\bar{\sigma}$ is the effective flow stress and $\bar{\varepsilon}$ is effective strain. Inserting Eqs. (2.23) into Eq. (2.22), the mean flow stress of the material is given by

$$
\sigma_{0}=\frac{1}{n+1} K\left(\frac{2}{\sqrt{3}} \ln \frac{t_{i}}{t_{f}}\right)^{n}
$$

According to Fig. 1, in all three zones each particle of the material undergoes different strains. The material in zone I has no strain-hardening, at the inlet shear boundary it has a strain due to discontinuity of velocity, in zone II the material undergoes strain due to deformation, after it, at the outlet shear boundary the material undergoes strain due to discontinuity of velocity and, finally, it exits from zone III with no deformation. By integrating the incremental strain along a stream line, the equivalent strain in the product is calculated. It has an angle of inclination $\theta$ to the axis in the deformation zone. At the shear surface, the engineering shear strain $\gamma_{S}$ is

$$
\gamma_{S}=\frac{|\Delta v|}{\dot{U}_{r}}
$$

and the effective strain on the shear boundary is

$$
\bar{\varepsilon}_{S}=\frac{1}{\sqrt{3}} \gamma_{S}
$$

At the inlet and outlet shear boundaries, the effective strains are, respectively

$$
\begin{aligned}
& \bar{\varepsilon}_{S_{1}}=\frac{1}{\sqrt{3}} \gamma_{S_{1}}=\frac{1}{\sqrt{3}} \frac{\left|\left(1-\rho_{i} \frac{\partial g}{\partial r} \frac{1}{1+(b / \alpha)^{2}}\right) \sqrt{1+\left(\frac{b}{\alpha}\right)^{2}} \sin \theta\right|}{\cos \theta+\frac{1}{g} \frac{\partial g}{\partial \theta} \sin \theta} \\
& \bar{\varepsilon}_{S_{2}}=\frac{1}{\sqrt{3}} \gamma_{S_{2}}=\frac{1}{\sqrt{3}} \tan \theta
\end{aligned}
$$

In the deformation zone, the effective strain is expressed by

$$
\bar{\varepsilon}_{d}=\int_{r_{f}}^{r_{i}} d \bar{\varepsilon}=\int_{r_{f}}^{r_{i}} \frac{d \bar{\varepsilon}}{d t} \frac{d t}{d L} d L
$$

where

$$
\frac{d \bar{\varepsilon}}{d t}=\dot{\bar{\varepsilon}}=\sqrt{\frac{2}{3}\left(\dot{\varepsilon}_{r r}^{2}+\dot{\varepsilon}_{\theta \theta}^{2}+2 \dot{\varepsilon}_{r \theta}^{2}\right)}=\frac{2}{\sqrt{3}} \sqrt{\dot{\varepsilon}_{r r}^{2}+\dot{\varepsilon}_{r \theta}^{2}} \quad \frac{d t}{d L}=\frac{1}{\sqrt{\dot{U}_{r}^{2}+\dot{U}_{\theta}^{2}}}
$$


where

$$
d L=d r \sqrt{1+r^{2}\left(\frac{d \theta}{d r}\right)^{2}}
$$

then

$$
\bar{\varepsilon}_{d}=\frac{2}{\sqrt{3}} \int_{r_{f}}^{r_{i}} \sqrt{\frac{\dot{\varepsilon}_{r r}^{2}+\dot{\varepsilon}_{r \theta}^{2}}{\dot{U}_{r}^{2}+\dot{U}_{\theta}^{2}}\left[1+r^{2}\left(\frac{d \theta}{d r}\right)^{2}\right]} d r
$$

Along the stream line, the equivalent strain in the deformed material is

$$
\bar{\varepsilon}(\theta)=\bar{\varepsilon}_{S_{1}}(\theta)+\bar{\varepsilon}_{d}(\theta)+\bar{\varepsilon}_{S_{2}}(\theta)
$$

After obtaining the total strain from Eq. (2.32) and substituting in the power law, one can rewrite the power terms in order to modify the upper bound method with considering the strainhardening. So the internal power, shear loss powers on $S_{1}$ and $S_{2}$ surfaces and the frictional power, respectively, are

$$
\begin{aligned}
& \dot{W}_{i}=\int_{V} \frac{\dot{\sigma \varepsilon}}{\sigma} d V=\frac{2}{\sqrt{3}} \int_{V} K(\bar{\varepsilon})^{n} \sqrt{\frac{1}{2} \dot{\varepsilon}_{i j} \dot{\varepsilon}_{i j}} d V \\
& \dot{W}_{S}=\int_{S_{v}} \tau|\Delta v| d S=\frac{1}{\sqrt{3}} \int_{V} K(\bar{\varepsilon})^{n}|\Delta v| d S \\
& \dot{W}_{f}=\int_{S_{f}} \tau|\Delta v| d S=\frac{m}{\sqrt{3}} \int_{V} K(\bar{\varepsilon})^{n}|\Delta v| d S
\end{aligned}
$$

\subsection{The required rolling torque}

By make use of the upper bound method, the externally supplied power is less than or equal to the sum of the powers described in the previous Sections. The total power $J^{*}$ is

$$
J^{*}=\dot{W}_{i}+\dot{W}_{S_{1}}+\dot{W}_{S_{2}}+\dot{W}_{f}
$$

The rolling torque $T$ is given by

$$
T=\frac{J^{*}}{\omega}
$$

where $T$ is the required rolling torque per unit width of the sheet. The rolling torque is a function of $b$ (shape factor) and $\rho_{N}$ (position of the neutral point). The shape factor $b$ determines the inlet shear boundary shape. The minimum value of rolling torque with respect to $b$ is the required torque for the rolling process in the upper bound analysis. Integrals appearing in the above equations do not have analytical solutions and they have been solved numerically with MATLAB software.

\section{Internal defects prediction criteria}

The external torque is a function of several parameters including roll radius, area reduction, friction factor and shape factor $b$. In the upper bound analysis, the minimum value of the external power with respect to $b$ is the required power for the rolling process. According to the 


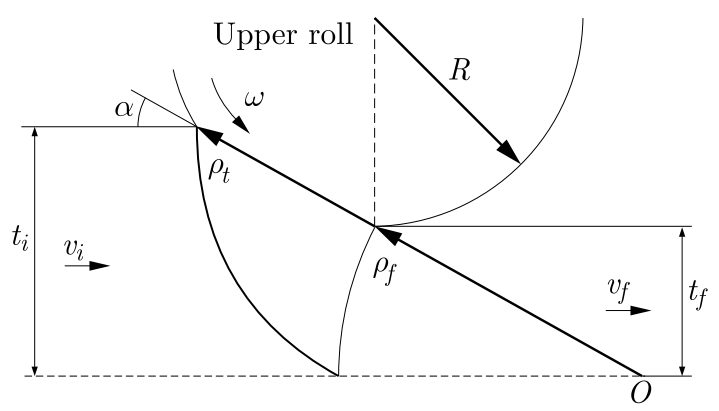

Fig. 2. Geometrical condition to initiate the internal defect

geometrical condition, for the critical value of shape factor $b\left(b_{c r}\right)$, the inlet shear boundary intersects the outlet shear boundary in the centerline, see Fig. 2.

In this study, it can be taken that

$$
r_{i}\left(\theta=0, \rho_{i}\right)=r_{f}\left(\theta=0, \rho_{f}\right)
$$

thus

$$
\rho_{i} \exp (-b)=\rho_{f}
$$

so, the critical value of $b$ can be obtained as

$$
b_{c r}=\ln \frac{\rho_{i}}{\rho_{f}}=\ln \frac{t_{i}}{t_{f}}
$$

So, if the shape parameter obtained from the optimization of the external power is equal or greater than the $b_{c r}$, the internal defects initiate.

\section{Results and discussion}

The flat rolling process has been analyzed using the proposed approach. In order to obtain numerical boundaries that can be applied to the prediction and prevention of the occurrence of internal defects in industry, finite element simulations using the proposed approach have been carried out for many combinations of reduction in the area and relative thickness. This means that no internal defects occur under this combination of process parameters. FEM simulations are conducted on the available commercial explicit/FEM software, ABAQUS, to verify the analytical model and study the effects of the upper bound method assumptions on the obtained results. Due to symmetry of the process, finite element meshes are generated on the upper half cross section of the sheet. The sheet is meshed by $2 \mathrm{D}$ plane strain, linear, four-noded CPE4R elements. The sheet model contains 460 elements. In this model, the rolls are modeled as rigid bodies. The rolls are rotated by a constant angular velocity about their axes. For verification of the theoretical study, the results of rolling torque are extracted from FEM simulations. In order to verify the validity of the upper bound approach for the flat rolling process presented in the previous Sections, the results obtained from the theoretical model are compared with the available experimental of data of Martins and Barata (1999) as well as with the results of finite element simulations. The calculation has been carried out under various rolling conditions and geometrical data utilized in the rolling analysis summarized in Table 1. During theoretical analysis and numerical simulations, $m$ is set at 0.3 for the contact surface between the roll and sheet, radius of the rolls is $R=79.375 \mathrm{~mm}$, and the flow stress for aluminum at room temperature is $\bar{\sigma}=50.3(1+\bar{\varepsilon} / 0.05)^{0.26} \mathrm{MPa}$. The comparisons between the computed results, 
Table 1. Geometrical data used for computations (Martins and Barata, 1999)

\begin{tabular}{|c|c|c|c|}
\hline Case & $\begin{array}{c}2 t_{0} \\
{[\mathrm{~mm}]}\end{array}$ & $\begin{array}{c}2 t_{f} \\
{[\mathrm{~mm}]}\end{array}$ & $\begin{array}{c}\text { Reduction } \\
{[\%]}\end{array}$ \\
\hline \hline 1 & 6.274 & 5.385 & 14.17 \\
\hline 2 & 6.274 & 4.902 & 21.86 \\
\hline 3 & 6.274 & 4.445 & 29.40 \\
\hline 4 & 6.274 & 4.115 & 34.41 \\
\hline
\end{tabular}

(a)

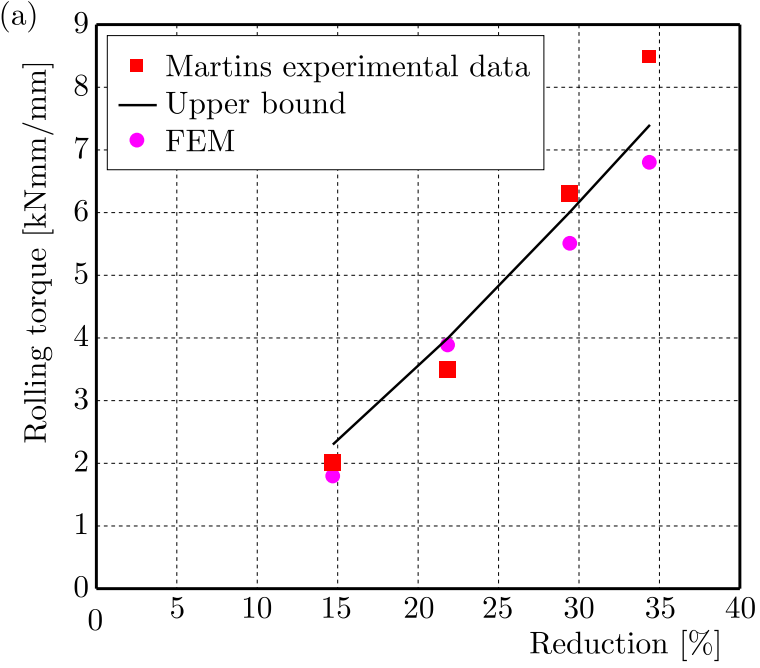

(b)

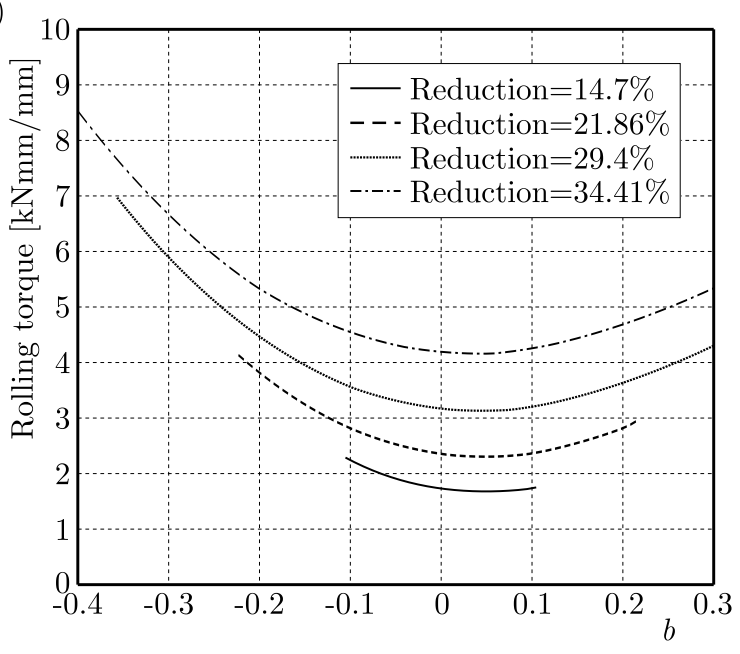

Fig. 3. (a) Comparison of analytical, FEM and Martins experimental data (Martins and Barata, 1999) of rolling torque (per unit of width) as a function of the percentage of reduction. (b) Variation of rolling torque per unit width versus parameter $b$

FEM simulation and the experimental values of Martins and Barata (1999) for the rolling torque as a function of the rolling reduction are shown in Fig. 3a. It is observed that the proposed velocity field leads to a computationally efficient procedure which gives a good agreement with the experimental data. From Fig. 3a, it can be seen that the calculated torques are basically in agreement with the measured ones. As expected, the predicted rolling torques are always greater than the experimental and FEM results, because the present theoretical values are the upper bound solutions. The reason for such discrepancies may be attributed to the assumption of rigid rolls as well as to difficulties in the modeling of friction in the contact surface between the rolls and the deforming sheet. It can be checked from Fig. 3a that the rolling torque increases with an increase in reduction. Figure $3 \mathrm{~b}$ shows variation of the rolling load versus the shape parameter $b$ for several reductions of the area according to Table 1 . The internal defects criterion is achieved after obtaining the shape parameter from both geometrical and analytical conditions and by comparing them. In Fig. 4, the velocity components obtained from the upper bound solution are compared with the FEM simulation results in $\theta=\alpha / 2$ in the deformation zone. The results show a good agreement between the upper bound data and the FEM results. It can be seen that the peripheral velocity components are very small with respect to the radial velocity components. To compare the numerical results with the experimental results of Ghos and Gardiner (2004), the analysis performed on Aluminum 6061-T6 whose mechanical and physical properties are shown in Table 2.

The flow stress for aluminum at room temperature is $\bar{\sigma}=410(\bar{\varepsilon})^{0.05} \mathrm{MPa}$ (Turczyn, 1996). The initial thickness is $t_{i}=10 \mathrm{~mm}$, roll radius $R=100 \mathrm{~mm}$, angular velocity $\omega=0.167 \mathrm{~s}^{-1}$ and friction factor $m=0.3$. Figure 5 shows the conditions of area reduction and relative thickness for preventing internal defects in Aluminum 6061-T6 for both conditions of strain-hardening 
(a)

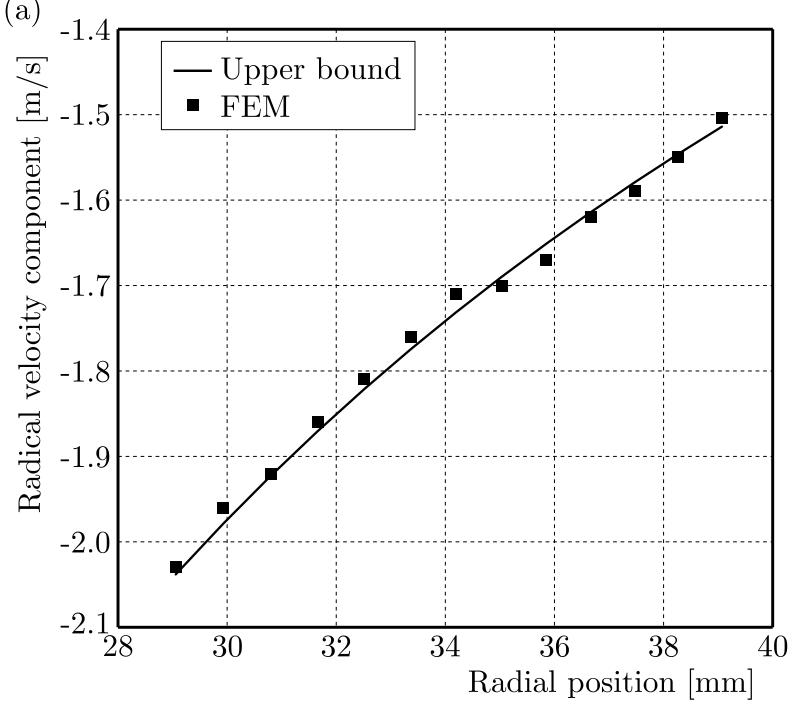

(b)

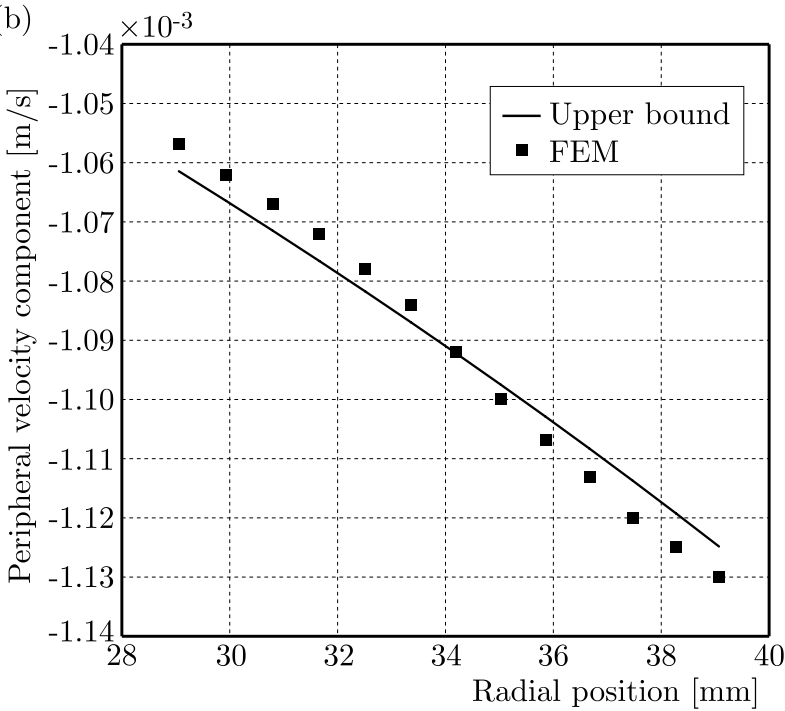

Fig. 4. Comparison of the upper bound and FEM of velocity components (a) radial and (b) peripheral velocity components for $30 \%$ reduction in the area

Table 2. Mechanical properties of Aluminum 6061-T6 (Turczyn, 1996)

\begin{tabular}{|c|c|c|c|c|c|}
\hline $\begin{array}{c}\text { Young's } \\
\text { modulus } \\
{[\mathrm{GPa}]}\end{array}$ & $\begin{array}{c}\text { Poisson's } \\
\text { ratio } \\
(\nu)\end{array}$ & $\begin{array}{c}\text { Yield } \\
\text { stress } \\
{[\mathrm{MPa}]}\end{array}$ & $\begin{array}{c}\text { Density } \\
{\left[\mathrm{kg} / \mathrm{m}^{3}\right.}\end{array}$ & $\begin{array}{c}\text { Strength } \\
\text { coefficient } \\
{[\mathrm{MPa}]}\end{array}$ & $\begin{array}{c}\text { Strain- } \\
\text {-hardening } \\
\text { exponent }\end{array}$ \\
\hline \hline 68.9 & 0.33 & 276 & 2700 & 410 & 0.05 \\
\hline
\end{tabular}

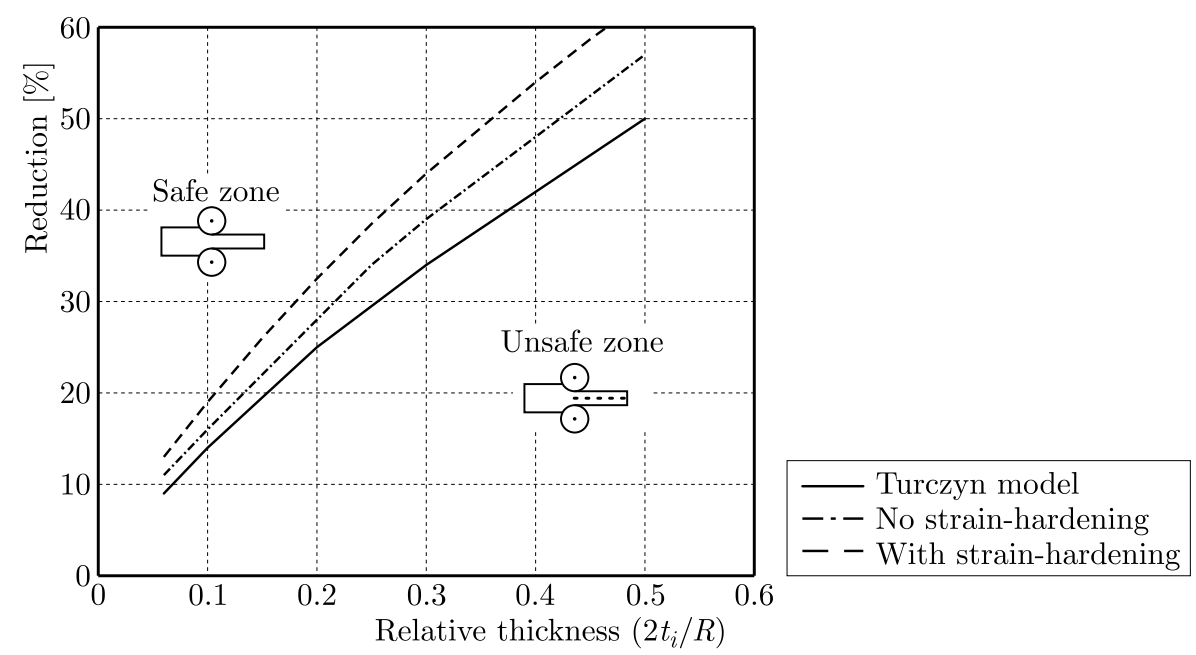

Fig. 5. Comparison of safe and unsafe zones predicted by the present model and the model by Turczyn (1996)

and perfectly plastic materials. Also, these results are compared with the experimental data by Turczyn (1996). It is observed that the safe domain is decreased by including the strain-hardening behavior of the material in the analysis.

Figure 6 a shows the velocity field in the flat rolling process with relative thickness $2 t_{i} / R=0.2$ and $40 \%$ reduction in area. The velocity field shows that in this case the internal defects do not occurr. In another model, see Fig. $6 \mathrm{~b}$, the reduction of the area is changed to $20 \%$ and the relative thickness is $2 t_{i} / R=0.4$, so that it locates in the unsafe zone. Also, Fig. $6 \mathrm{~b}$ shows that the internal defects occurr. 
(a)

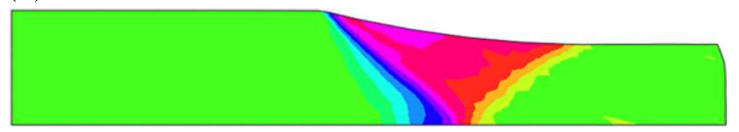

(b)

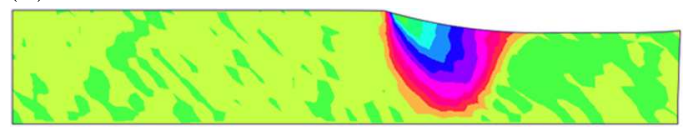

Fig. 6. Deformation zone, (a) for $2 t_{i} / R=0.2$ and $40 \%$ reduction in the area, (b) for $2 t_{i} / R=0.4$ and $20 \%$ reduction in the area

Figure 8 shows the effect of friction factor on internal defects initiation situations for Aluminum 6061-T6. It can be seen that with an increase in the friction factor, the safe zone size decreases. The effect of the strain-hardening exponent on the inlet shear boundary is shown in Fig. 8. From this figure, it can be noticed that with an increase in the strain-hardening exponent, the intersection point of the inlet shear boundary with the axis of symmetry moves towards the outside of the rolls, and the tendency of central bursting defects is decreased. Also, the criterion applied for different strain-hardening exponents is illustrated in Fig. 9a. This figure shows that with an increase in the strain-hardening exponent value, the safe domain increases. Figure $9 \mathrm{~b}$ shows the effect of strain-hardening exponent on the rolling torque for the upper bound approach and the FEM. This figure illustrates that by increasing the strain-hardening exponent, the rolling torque decreases. Figure $9 \mathrm{~b}$ also shows that the theoretically predicted rolling torque is higher than that from the FEM results, which is due to the nature of the upper bound theory.

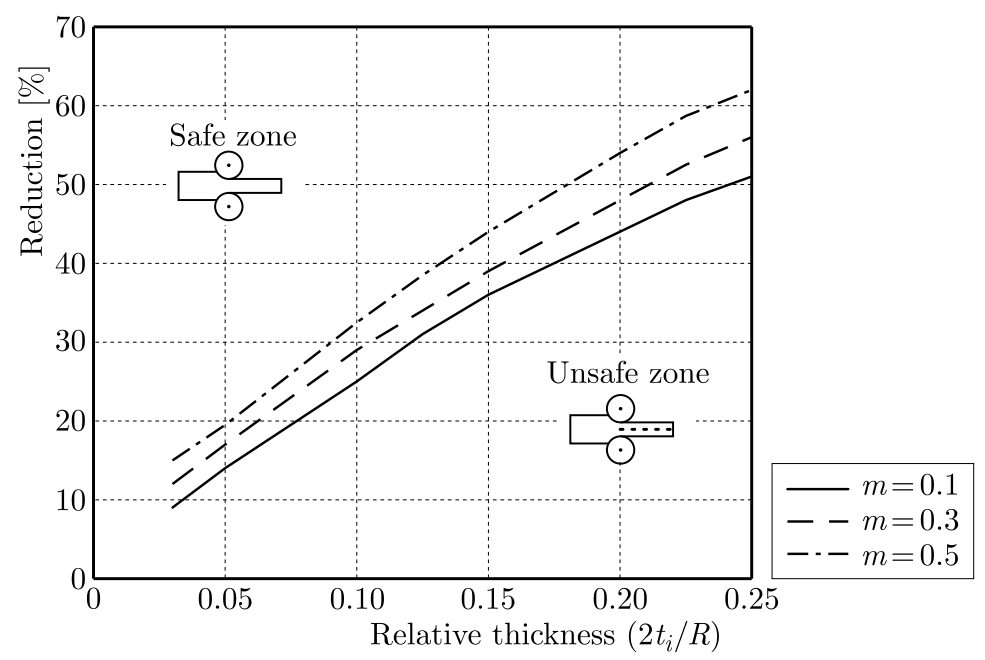

Fig. 7. Effect of the friction factor on size of the safe and unsafe zones

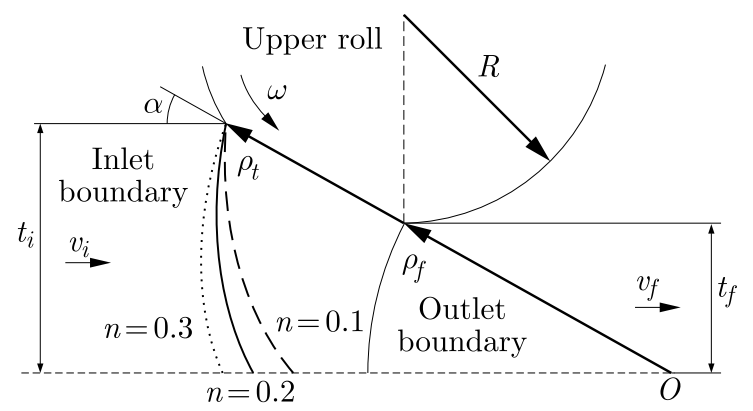

Fig. 8. Effect of the strain-hardening exponent on the inlet shear boundary 
(a)

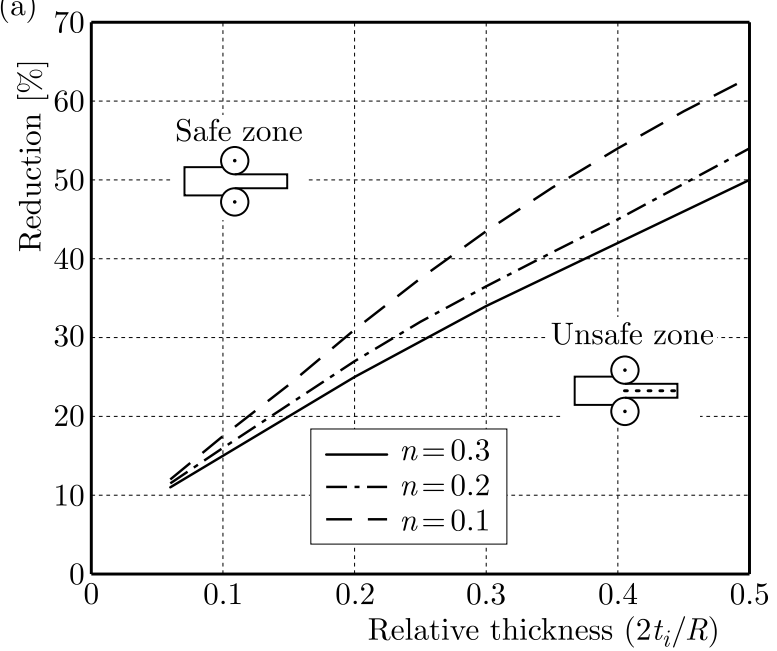

(b)

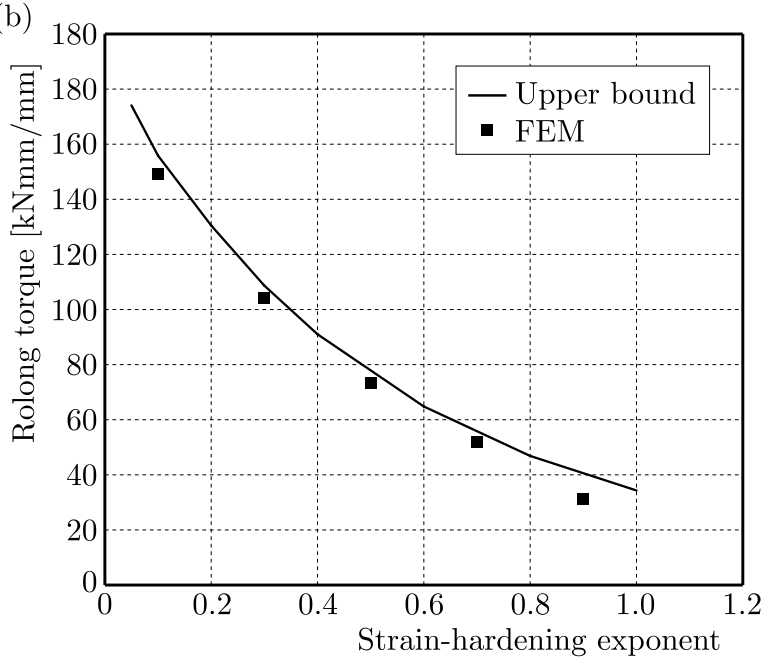

Fig. 9. Effect of the strain-hardening exponent (a) on size of the safe and unsafe zones and (b) on the rolling torque for $30 \%$ reduction in the area

\section{Conclusions}

In this paper, an analytical approach based on the upper bound method, is presented for prediction of rolling torque and occurrence of internal defects in the flat rolling process. The advantages of the presented criterion are the generality of the proposed inlet shear boundary and a new kinematically admissible velocity field. In addition, the presented criterion predicts central bursting defects in simpler mathematical equations than in other criteria, and the effect of the strain hardening exponent of the sheet material on the rolling torque and prediction of internal defects are investigated. It has been concluded that internal defects are affected primarily by the strain-hardening exponent. By increasing the strain-hardening exponent, the rolling torque and the possibility of internal defects decreases. By increasing the strain-hardening exponent, the safe zone size is increased and the internal defects occur in thick sheets with a small reduction. Criteria curves for the safe domains are presented for a wide range of process variables. By using these criteria in the rolling practice, it has become possible to predict necessary rolling conditions in order to avoid internal defects.

\section{References}

1. Avitzur B., Van Tyne C., Turczyn S., 1988, The prevention of central bursts during rolling, Journal of Engineering for Industry, 110-173

2. Caoa T., Bobadillab C., Montmitonneta P., Bouchard P., 2015, A comparative study of three ductile damage approaches for fracture prediction in cold forming processes, Journal of Materials Processing Technology, 216, 385-404

3. Deng W., Zhao D., Qin X., Du L., Gao X., Wang G., 2009, Simulation of central crack closing behavior during ultra-heavy plate rolling, Computational Materials Science, 47, 439-447

4. Dogruoglu A., 2001, On constructing kinematically admissible velocity fields in cold sheet rolling, Journal of Materials Processing Technology, 110, 287-299

5. Dyja H., Pietrzyk M., 1983, On the theory of the process of hot rolling of bimetal plate and sheet, International Journal of Mechanical Working Technology, 8, 309-325

6. Ghosh S., Li M., Gardiner D., 2004, A computational and experimental study of cold rolling of aluminum alloys with edge cracking, Transactions of the ASME, 126, 74-82 
7. Haghighat H., Amjadian P., 2013, A generalized upper bound solution for extrusion of bimetallic rectangular cross-section bars through dies of any shape, Journal of Theoretical and Applied Mechanics, 51, 105-116

8. Haghighat H., Parghazeh A., 2017, An investigation into the effect of strain hardening on the central bursting defects in rod extrusion process, The International Journal of Advanced Manufacturing Technology, accepted for publication

9. Haghighat H., SaAdati P., 2015, An upper bound analysis rolling process of non-bonded sandwich sheets, Transaction of Nonferrous Metals Society of China, 25, 1605-1613

10. Martins P., Barata M., 1999, Upper bound analysis of plane strain rolling using a flow function and the weighted residuals method, International Journal for Numerical Methods in Engineering, 44, 1671-1683

11. Misicko R., Kvackaj T., Vlado M., Gulova L., Luptak M., Bidulska J., 2009, Defects simulation of rolling strips, Materials Engineering, 16, 7-12

12. Prakash R., Dixit P., 1995, Steady state plane strain cold rolling of a strain-hardening material, Journal of Materials Processing Technology, 52, 338-358

13. RAJAK S., REDDy N., 2005, Prediction of internal defects in plane strain rolling, Journal of Materials Processing Technology, 159, 409-417

14. Serajzadeh S., Mahmoodkhani Y., 2008, A combined upper bound and finite element model for prediction of velocity and temperature fields during hot rolling process, International Journal of Mechanical Sciences, 50, 1423-1431

15. Takuda H., Kyoto H., Lippmann H., Kokado J., 1989, Upper bound approach to plane strain strip rolling with free deformation zones, Ingenieur-Archiv, 59, 274-284

16. Turczyn S., 1996, The effect of the roll-gap shape factor on internal defects in rolling, Journal of Materials Processing Technology, 60, 275-282

17. Turczyn S., Pietrzyk M., 1992, The effect of deformation zone geometry on internal defects arising in plane strain rolling, Journal of Materials Processing Technology, 32, 509-518 